\title{
Gait Event Detection in Controlled and Real-life Situations: Repeated Measures from Healthy Subjects
}

\author{
Joana Figueiredo, Paulo Félix, Luís Costa, Juan C. Moreno, Senior Member, IEEE, Cristina P. Santos, \\ Senior Member, IEEE
}

\begin{abstract}
A benchmark and time-effective computational method is needed to assess human gait events in real-life walking situations using few sensors to be easily reproducible. This study fosters a reliable gait event detection system that can operate at diverse gait speeds and on diverse real-life terrains by detecting several gait events in real-time. This detection only relies on the foot angular velocity measured by a wearable gyroscope mounted in the foot to facilitate its integration for daily and repeated use. To operate as a benchmark tool, the proposed detection system endows an adaptive computational method by applying a finitestate-machine based on heuristic decision rules dependent on adaptive thresholds. Repeated measurements from eleven healthy subjects $(28.27 \pm 4.17$ years) were acquired in controlled situations through a treadmill at different speeds (from $1.5 \mathrm{~km} / \mathrm{h}$ to $4.5 \mathrm{~km} / \mathrm{h}$ ) and slopes $(0 \%$ to $10 \%)$. This validation also includes heterogenous gait patterns from nine healthy subjects $(27 \pm 7.35$ years) monitored at three self-selected paces (from $1 \pm 0.2 \mathrm{~m} / \mathrm{s}$ to

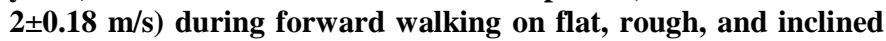
surfaces and climbing staircases. The proposed method was significantly more accurate $(p>0.9925)$ and time-effective $(<30.53 \pm 9.88 \mathrm{~ms}, p>0.9314)$ in a benchmarking analysis with a state-of-the-art method during 5657 steps. Heel Strike was the gait event most accurately detected under controlled (accuracy of $100 \%$ ) and real-life situations (accuracy $>96.98 \%$ ). Misdetection was more pronounced in Middle Mid-Swing (accuracy $>90.12 \%$ ). The lower computational load, together with an improved performance, makes this detection system suitable for quantitative benchmarking in the locomotor rehabilitation field.
\end{abstract}

Index Terms - Human gait analysis, real-time gait event detection, adaptive computational methods, wearable inertial sensors, daily locomotion activities

\section{INTRODUCTION}

$\mathrm{G}$ AIT event detection can potentially be applied in the rehabilitation field, namely, in the design of personalized gait therapies that tune therapeutic assistance in accordance to the patient-specific needs and attempt to foster a more efficient functional motor recovery [1]-[5].

Different motion capture systems have been used to assess human gait events. Most commonly, this analysis is conducted in a motion analysis laboratory with force platforms and optical

This work has been supported by the FCT - Fundação para a Ciência e Tecnologia - with the reference scholarship SFRH/BD/108309/2015, with the reference project UID/EEA/04436/2013, and by FEDER funds through the COMPETE 2020 - Programa Operacional Competitividade $e$ Internacionalização (POCI) - with the reference project POCI-01-0145FEDER-006941 and supported by grant RYC-2014-16613 by Spanish Ministry of Economy and Competitiveness. motion systems. Nevertheless, these motion capture systems are non-portable [6], operate only in controlled environments [7], and are consequently not optimal to analyze consecutive gait cycles for long-term mobility scenarios [4].

Current research suggests that there is a need for assessing human locomotion in non-structured conditions. Technological advances in wearable sensors have approached this issue. Force-based systems, such as foot-switches or force sensitive resistors (FSRs), are generally considered the gold standard for detecting gait events [8]. However, these sensors (i) are prone to mechanical failure [1], (ii) can be unreliable when used by patients with drop-foot due to their shifting weight during standing [3], [9]-[12], and (iii) do not provide any information regarding the sub-phases of the swing phase [9], [12].

To overcome some of the inherent limitations of force-based sensors, recent studies have explored the potentialities of inertial sensors, particularly isolated accelerometers [10], [11], [13], [14], isolated gyroscopes [6], [15]-[19], and inertial measurement units (IMUs) [2], [4], [9], [20]-[23] for real-time gait event detection. Nonetheless, heel-strike vibrations [9], [14] may limit the use of accelerometers compared to gyroscopes. According to systematic review of Taborri et al. [1], gyroscopes provide better performance than other inertial quantities for monitoring human gait. There is no consensus regarding the best location for the gyroscope on the user's body. Commonly, gyroscopes placed on the shank [4], [6], [9], [15][18], [23] and on the foot [2], [4], [10], [11], [19], [20], [24] lead to the more reliable gait event detection. However, Aung et al. [11] demonstrated better performance with the sensor located on the foot rather than ankle or shank.

Most of the available computational methods that use measurements from the gyroscope for gait segmentation are based on the definition of heuristic thresholds through a rulebased finite state machine (FSM) [4], [6], [10], [15], [16], [18], [23]. The threshold-based FSM proposed by Catalfamo et al. [6] was able to detect $98 \%$ of HS and TO events performed by healthy children in indoor and outdoor inclined and flat scenarios. Kotiadis et al. [22] showed that using the gyroscope and accelerometer data as inputs of the FSM resulted in the

Joana Figueiredo (id6003@alunos.uminho.pt), Paulo Félix (paulo.felix.oliveira@gmail.com), Luís Costa (luisorlandocosta2@gmail.com) and Cristina P. Santos (cristina@dei.uminho.pt) are with Center for MicroElectroMechanical Systems (CMEMs), University of Minho, Guimarães, 4800-058, Portugal.

Juan C. Moreno (jc.moreno@csic.es) is with Neural Rehabilitation Group, Spanish National Research Council, Cajal Institute, Madrid, Spain. 
proper detection of $\mathrm{HS}, \mathrm{TO}$, and $\mathrm{HO}$ events on flat surface and staircase walking. Furthermore, Storm et al. [23] demonstrated that the threshold-based FSM can accurately segment (accuracy of $100 \%$ ) the HS and TO events performed by healthy subjects free-walking in an indoor and outdoor urban environment.

The increased popularity of the FSM is mainly explained by its low computational demand and easy application [16]. Nevertheless, it has been reported that its performance can be affected by the high inter-subject [19] and inter-step variability [20]. To surpass the limited generalization when processing new datasets, previous studies [5], [9], [25], [26] introduced an updating layer for tailoring the thresholds endowed in the heuristic rules with the most recent state of the gait pattern.

Nonetheless, to the authors' knowledge, the assessments of gait events that have been proposed in the literature have been conducted in controlled environments (i.e., trials performed on treadmill or static walkways) and level-ground walking [2], [3], [5], [10], [14], [15], [19], [27]. When applied to real-life situations, the gait analyses reported in the literature have mostly been restricted to two or three gait events [6], [8], [14], [16], [18], [23]. Furthermore, evaluations have reported few repeated measures with different subjects, which is a problematic for evaluation of the reproducibility of the proposed computational methods under real-life applications. The absence of a quantitative computational benchmark for the assessment of human gait events is a concern in the rehabilitation research community.

Considering this current state-of-the-art, there is a need to find time-effective, reliable, inter-subject and inter-step versatile computational solutions that describe human gait in both controlled and real-life situations using few wearable sensors in an attempt to be easily reproducible under different contexts. In this sense, this study aims to provide a reliable gait event detection system that is able to operate during different daily locomotion activities, and is able to detect, in real-time, several human gait events: Heel Strike (HS), Foot Flat (FF), Middle Mid-Stance (MMST), Heel-Off (HO), Toe-Off (TO) and Middle Mid-Swing (MMSW). The detection of these events constitutes a novel state-of-the-art contribution, as analyses have typically been centered on the detection of HS, TO and MMSW events. As human gait is quite dynamic in reallife, the challenge of this work has been to develop and validate the performance of a tool that is suitable for varying gait speeds, changing surfaces and varying surface inclinations, even when the healthy subject is walking barefoot or with footwear. The adaptability to different real-life walking conditions represents an additional contribution to the current challenges. Few studies have performed gait event detection in real-life situations; Catalfamo et al. [6] considered inclined surfaces (indoor and outdoor environments) while Formento et al. [16] included trials in staircases. To ensure such adaptability, our approach extends to previous teamwork [26] by applying a robust FSM triggered by adaptive thresholds in heuristic decision rules.

Moreover, based on the reliable performance identified by Taborri et al. [1], this computational method only uses the single-axis of a wearable gyroscope (compact and low power consumption sensor) placed on the user's feet. We sought to minimize the number of sensors in an attempt to provide a lowcost solution that was easily reproducible in real-life and did not disturb the human motion. The developed method demands a low computational load that allows for its implementation in embedded processing systems and to reduce the system latency. Heterogenous and repeated measures from healthy subjects were used to investigate the timing and precision performance of the adaptive computational method in comparison to those of force sensors. These measures were also used to perform a direct comparison between the proposed rule-based method and the machine learning method proposed by Mannini [19] in the detection of HS, FF, HO, and TO events. To the best knowledge of the authors, no other study in the literature has developed a real-time gait segmentation strategy based only on a single-axis of a gyroscope that is able to detect several gait events (HS, FF, MMST, HO, TO, and MMSW) across different real-life situations. Lastly, the article proposes a detection tool that acts as a quantitative and computational benchmark for assessing human gait events, which is currently not available.

\section{METHODS}

\section{A. Wearable Sensory System}

To fulfill the portability and real-time constraints, the proposed gait event detection system relies on a gyroscope embodied on the InertialLAB system to measure the angular velocity at $100 \mathrm{~Hz}$ and a high-performance microcontroller to run the adaptive computational method (STM32F4-Discovery with an AMR® Cortex® -M4 32-bit core).

InertialLAB (Fig.1) is a wearable inertial sensory system that was designed for ambulatory human gait sensing in diverse walking situations, such as those tested in this study. It includes two inertial units placed on the instep of each foot, as illustrated in Fig. 1, and a central processing unit. Each inertial unit is based on the MPU6050, a low-cost IMU that combines a tridimensional accelerometer $( \pm 8 \mathrm{~g})$ and a tridimensional gyroscope $( \pm 2000 \%$ ) for the acquisition of feet kinematic data. For data acquisition, we selected the STM32F4-Discovery microcontroller with an attached USB flash drive to store the collected data. We used the I2C communication protocol to connect each inertial unit to the central unit using USB cables (easy plug and unplug solution for real-life gait sensing). InertialLAB is powered by a standard $2000 \mathrm{mAh}$ power-bank.



Fig. 1. Set-up of the InertialLAB and its location in a healthy user. 
For the purposes of ergonomics, easy donning and doffing, and portability, each inertial unit is fixed in cases and attached to the feet by adjustable ribbons (see Fig. 1). This procedure also allows for secure fastening to the body part with minimum sensor motion relative to the foot, thus avoiding fluctuations in the IMU angular velocity signal. The USB cables are in spiral form to meet the anthropometry requirements of covering $10^{\text {th }}$ to- $90^{\text {th }}$ percentile of male/female target population.

To ensure the repeatability of the sensor's alignment, the sensor was always positioned by the same person, who carefully positioned a visual mark on the sensor cases so that the gyroscope was aligned with the navicular and/or cuneiform bones (instep of the foot). This gyroscope's alignment enables the direct measurement of the foot angular velocity along the sagittal plane (the sensor's z-axis, as depicted in Fig. 1).

\section{B. Adaptive Computational Method}

This section focuses on the design and implementation of the adaptive gait segmentation algorithm considering the ground surfaces and speed changes.

\section{Definition of heuristic decision rules}

First, we verified that the foot angular velocity presented a constant waveform on level-ground, inclined surfaces and staircases (see Appendix I). Thus, heuristic rules can be designed for the reliable detection of the human gait events. Previous studies have shown that this kinematic feature aligned with the sagittal plane is more versatile for real-time gait detection across different ground surfaces [1], [28].

For the definition of the heuristic decision rules, we segmented the angular velocity signal into six moments that correspond to the six gait events to be assessed: HS, FF, MMST, HO, TO, and MMSW. To determine the exact moments of HS, $\mathrm{HO}$ and TO events, two FSRs were placed on the heel and toe (see Fig. 6). HO and TO events were set as the decreasing moment (when the FSR signal decreases by $70 \%$ relative to its maximum) of the heel and toe FSR signal, respectively, whereas the HS event consisted of the increasing instant (when the FSR signal is $70 \%$ higher than its minimum) of the heel FSR. We set the ground truths for FF, MMST, and MMSW events based on direct visual inspection of the video-based angular velocity with the IMU angular velocity, both were overlapped and synchronized by overlay tools of an opensource tracker. Fig. 2 depicts each gait event associated with the foot angular velocity signal.

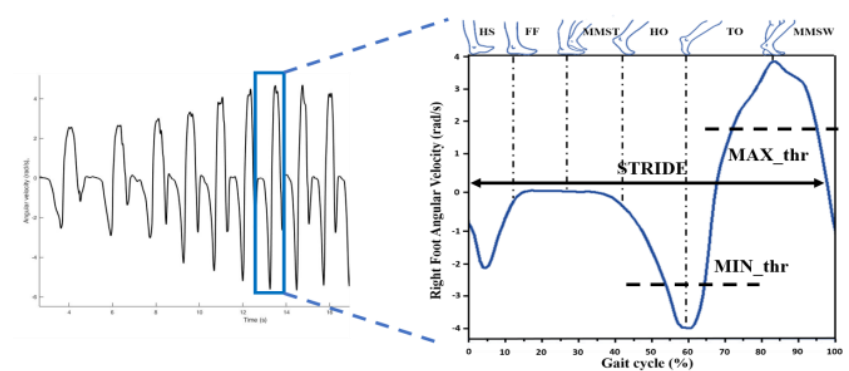

Fig. 2. Angular velocity of the right foot along the sagittal plane (sensor's zaxis) (continuous line) and representation of six human gait events (HS, FF, MMST, HO, TO, and MMSW) during one gait cycle performed by a healthy subject.
We also considered the literature regarding the gyroscope signal in the sagittal plane, as follows. For the FF and MMST events, the foot is flat to the ground, and consequently, the angular velocity is almost steady at $0 \%$ until the $\mathrm{HO}$ event [7], [29]. Sabatini et al. [7] and Pappas et al. [29] stated that the HO event occurs, approximately, after the zero-crossing of the gyroscope signal. According to [7], [29], [30], the gyroscope signal reaches the global minimum at the TO event. Studies [7], [20], [30] also report that during the swing phase, the peak value of the main feature appearing in the gyroscope signal is a rather broad positive pulse, which occurs at the moment of mid-swing (i.e., MMSW event). These results are in accordance with the gait events identified in this study and presented in Fig. 2.

We defined six different decision rules for the detection of each gait event, as indicated in Table I. The decision rules are based on curve tracing techniques, such as adaptive thresholds crossing, local extrema detection (i.e., maximum and minimum angular velocity), and the evaluation of signal derivatives. Generically, we defined: HS as the first instant in which the angular velocity is within a range empirically determined to be close to the null angular velocity $\left(H S \_t h r_{\text {mean }} \pm H S \_t h r_{\text {std }}=-0.5\right.$ \pm 0.05 ) after the maximum value has occurred; FF was defined as when the signal becomes approximately constant ( $n$ samples with the $1^{\text {st }}$ derivative almost null) after the detection of the $1^{\text {st }}$ minimum; MMST was defined as $n$ samples after FF occurred ( $n$ corresponds to the duration of the last valid MMST); HO was defined as when the velocity becomes negative after a constant period; TO was the $2^{\text {nd }}$ minimum detected by an adaptive threshold ( $M I N_{t h r}$ in Fig. 2), and MMSW was determined as the maximum detected above an adaptive threshold $\left(M A X_{t h r}\right.$ in Fig. 2 ). The rules also have a condition that depends on stride time (STRIDE_TIME in Fig. 2), which establishes adaptative intervals where the events shall occur and increases the robustness of the algorithm to changes in gait speed. The adaptive stride time computation will be presented next.

\section{Adaptability and Finite State Machine}

Our approach considers adaptability as a pivotal future for developing a benchmark method for gait analysis. To address the variation in gait pattern, the proposed method inspects changes in the duration and amplitude of angular velocity since both parameters can change with variations in gait speed. This information is used to adjust the adaptive thresholds of decision rules (MAXthr and MINthr) and the intervals in which the events must occur (conditions dependent on STRIDE_TIME).

Fig. 3 shows the flow chart of the proposed computational method, which is formed by six steps executed via STM32F4Discovery in each interaction at $100 \mathrm{~Hz}$ (the same frequency as the gyroscope data acquisition). Initially, the parameters (e.g., STRIDE_TIME, MAXthr, and MINthr) used in these steps were set a priori based on an exhaustive empirical inspection. The computational method only starts the detection of gait events after the occurrence of the maximum peak of angular velocity.

The algorithm starts with the acquisition of angular velocity in the sagittal plane through the gyroscope of InertialLAB. Since the algorithm uses real-time peak detection, it was necessary to smooth the gyroscope data through a digital $1^{\text {st }}$ 
order low-pass filter (exponential smoothing). Posteriorly, the filtered sample was analyzed in 3 different stages to make the FSM adaptable for different real-life walking situations. The first stage determines the $1^{\text {st }}$ derivative by detecting when the velocity increases (positive signal), decreases (negative signal) or becomes approximately zero.

The second stage covers the minimum/maximum calculation, which is used to detect HS (after maximum), MMSW (maximum), FF (after $1^{\text {st }}$ minimum) and TO ( $2^{\text {nd }}$ minimum), given their dependency on the local extrema. To ensure a robust local extrema detection (i.e., the detection of maximum and minimum angular velocity), in real-time, we considered different criteria based on adaptive thresholds and the signal derivative evaluation (gyroDER in Fig. 3). As illustrated in Fig. 3, a maximum angular velocity was only detected when the angular velocity was higher than the adaptive threshold $\left(M A X_{t h r}\right)$, the previous signal derivative is a positive value and the current signal derivative is negative soon after the gyroDER is approximately zero (less than $0.01 \mathrm{rad} / \mathrm{s}$ - value set empirically). We set the last condition to a value near zero instead of a zero value (theoretically a constant derivative that corresponds to local extrema) to address the signal fluctuations or noise around the detection of local extrema, as shown in Fig.

TABLE I 4.a). These fluctuations can change the signal derivative, and consequently, incorrectly detect local extrema. This allows for detection only major variations, which are usually associated with local peaks. We applied a similar approach to detect the angular velocity minima, using the adaptive threshold $\left(M I N_{t h r}\right)$.

As indicated in Fig. 3, the Max/Min detection stage also updates the MAXthr and MINthr thresholds using the three previous valid strides. MAXthr and MINthr were defined as $60 \%$ of the mean value of the 3 previous detected maxima and minima, respectively. For all walking conditions, the MAXthr and MINthr thresholds were initialized to $0.7 \mathrm{rad} / \mathrm{s}$ and $-2 \mathrm{rad} / \mathrm{s}$, respectively. Both the initial values and the percentages defined to update the adaptive thresholds were empirically found after an exhaustive inspection of the angular velocity from distinct gait patterns. This constitutes an adaptability point that allows for the proper behavior of the proposed computational method under changes in gait speed. It is important to note that if there are not three valid strides, the thresholds are updated using a prior valid stride until this criterion is met. In the beginning, the computational method uses the thresholds set as initial conditions.

DECISION RULES WITH ADAPTIVE THRESHOLDS IN GENERIC FORM

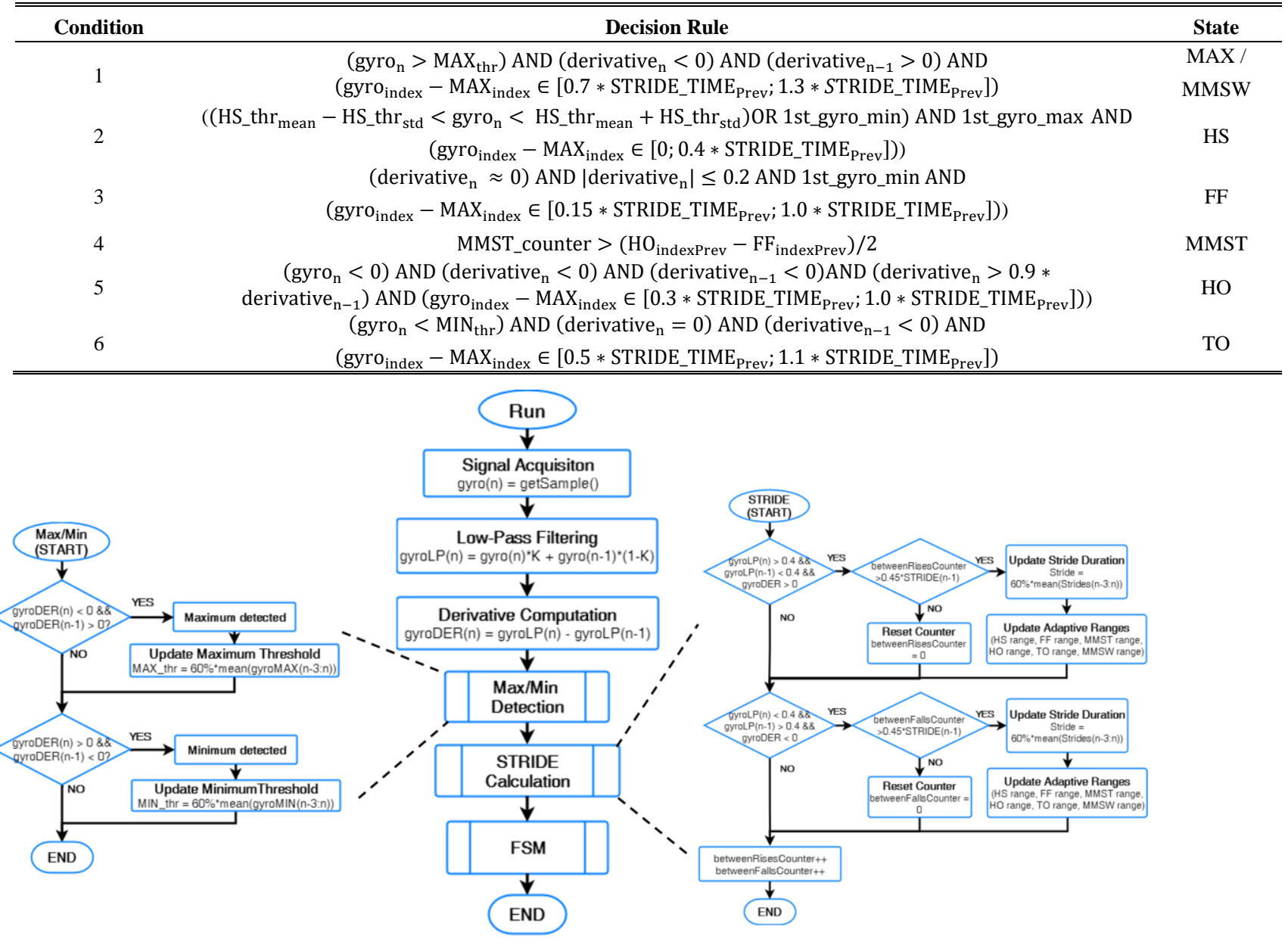

Fig. 3. Flow chart of the proposed adaptive computational method. 


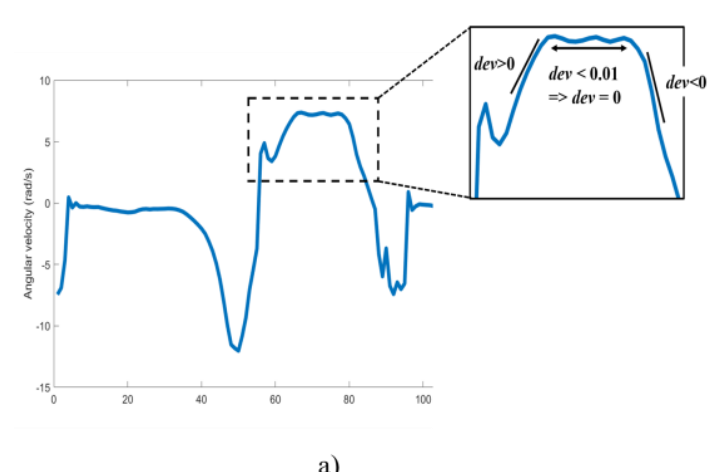

a)

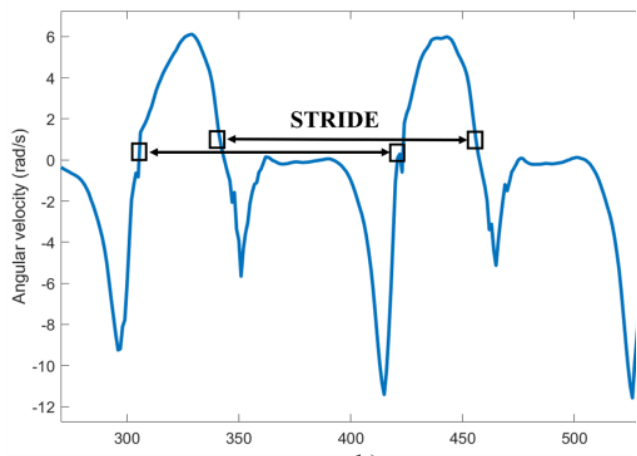

b)

Fig. 4. Foot angular velocity: a) derivative ( $(e v)$ behavior in the maximum detection; b) stride time calculation considering rising or declining periods.

A second adaptability point occurs in the STRIDE_TIME calculation stage. The current stride duration is used to adaptively compute STRIDE_TIME parameter based on the last three valid strides. For the first three strides, the initial conditions are used until a valid STRIDE_TIME is obtained. To compute a valid stride, we looked for rising periods (as shows Fig. 4.b)) where the angular velocity was higher than $0.4 \mathrm{rad} / \mathrm{s}$ with a positive derivative, and at this moment a duration at least $45 \%$ of the previous stride has passed (betweenRisesCounter in Fig. 3). Alternatively, a valid stride can be determined using the same approach with declining periods (in this case the parameter betweenFallsCounter in Fig. 3 would be considered). Both approaches update the STRIDE_TIME.

As indicated previously, STRIDE_TIME is used in the adaptive computational method to establish the adaptive ranges where the events must occur. These statistical decision limits, listed in Table I, were determined by trial and error after an empirical analysis that included multiple walking conditions. For each decision rule, an initial condition was set based on the percentage of the gait cycle assigned in the literature for a healthy gait [31]. Subsequently, these initial conditions were empirically tuned, resulting in the multiplication factors listed in Table I. The tuning procedure ended when the proposed algorithm reached similar results (i.e., error rate up to $20 \%$ ) to those of the ground truths. This strategy tailors the algorithm to properly operate at distinct gait speeds and allows the FSM to restart when an event is not detected (exit condition - E). It is worth mentioning that with this stage, in addition to assess the human gait events, the proposed computational method is able to determine the real-time stride duration.

The last stage implements the FSM through the switch statement presented in Fig. 5. The proposed FSM presents six states, one for each gait event (MAX/MMSW, HS, FF, MMST, $\mathrm{HO}, \mathrm{TO}$ ), and two additional states (default state - DEF, and reset state - R). The decision rules defined in Table I (1-6) and the exit condition (E) are used to trigger the state transitions. As indicated in Fig. 5, the $1^{\text {st }}$ state to run is the R state. Here, all variables are reset and the initial conditions (empirically tuned) are set. Next is a transition to the DEF state. The FSM only leaves the DEF state when rule 1 is true (maximum), transiting to MAX/MMSW. Note that this rule only allows for the transition to the MAX state in the first detection. In the remaining situations, it detects the MMSW since the maximum of the angular velocity corresponds to this event. The FSM is also adaptive in the calculation of the threshold for the MMST (MMST_counter).

At last, the developed tool can also address situations in which the user stands for a period of time without walking. In this case, the information from previous steps will not be used since the gait pattern was changed. Thus, the algorithm resets after a pre-defined time (at least 5.0* STRIDE_TIME). When this occurs, this computational method sets all empirical parameters to the values defined in the initial conditions.

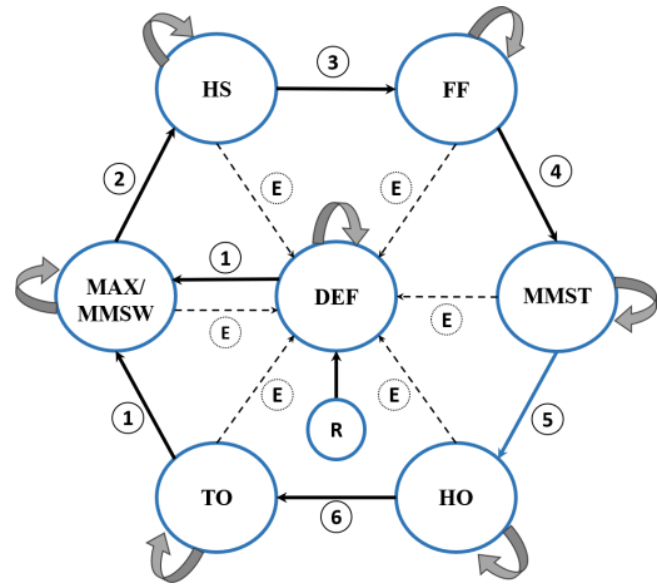

Fig. 5. FSM used to detect the gait events.

\section{Validation of gait event detection system}

We validated the adaptive computational method using repeated measures of healthy gait patterns recorded in controlled and real-life situations, as depicted in Fig. 6. Twenty subjects were involved in two protocols, one for each condition. The subjects signed a written informed consent to participate in this study and were randomly divided into the two protocols.

The detected and reference gait events were stored as text files on a USB flash drive attached to InertialLAB for a subsequent validation through Matlab® (2016a, The Mathworks, Natick, MA, USA). To establish the ground truths, we used a reference measurement system to detect true gait events at $100 \mathrm{~Hz}$. To achieve this, we integrated two FSRs in the heel and toe (see Fig. 6) that enabled the identifications of HS, HO and TO events, as previously described. The FF, 
MMST, and MMSW events were found through visual inspection by a gait analysis expert and based on the information reported in the literature [7], [20], [30].

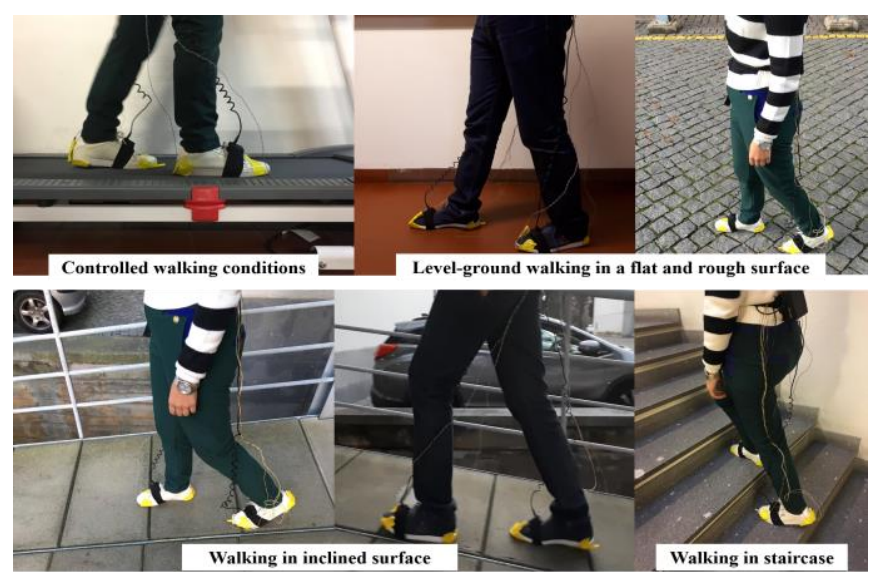

Fig. 6. Validation of the gait event detection system under controlled and reallife walking conditions (flat and rough level-ground, inclined surfaces and staircases). The users wore two units of InertialLAB (instep of the foot) and two FSRs attached to heel and toe by a yellow rubber strap.

\section{Controlled walking situations}

We validated the proposed algorithm under controlled walking situations to test the effect of variations in ground surface and gait speed. We included 11 healthy volunteers (7 males and 4 females). The subjects presented an average age of $28.27 \pm 4.17$ years old, the height of $1.70 \pm 0.08 \mathrm{~m}$, and weight of $69 \pm 12.02 \mathrm{~kg}$. The participants were randomly divided into barefoot (6 subjects) and footwear conditions ( 5 subjects).

The participants conducted walking experiments on an instrumented split-belt treadmill at different speeds (1.5, 2.5, 3.5 , and $4.5 \mathrm{~km} / \mathrm{h})$ and slopes $(0 \%, 5 \%$, and $10 \%)$. All combinations of gait speed and incline were randomly performed. We asked the subjects to perform 3 trials of 30 seconds per condition. Furthermore, the participants were instructed to conduct walking trials at variable speeds to approximate a real-life environment. In this case, the subjects walked for 60 seconds and changed gait speed every 20 seconds according to the provided instructions (increasing from 1.5 $\mathrm{km} / \mathrm{h}$ to $4.5 \mathrm{~km} / \mathrm{h}$ and decreasing from $4.5 \mathrm{~km} / \mathrm{h}$ to $2.5 \mathrm{~km} / \mathrm{h}$ ). To provide reliable results, the acceleration period was not considered in the detection of gait events, except in the trials where the speed was variable.

\section{Real-life walking situations}

The algorithm validation was extended to real-life situations to evaluate human locomotion in different conditions. For this matter, we included 9 healthy subjects ( 6 males and 3 females), who wore their own sports-shoes. The participants' mean age is $27 \pm 7.35$ years old, and they presented a height of $1.70 \pm 0.12 \mathrm{~m}$ and weight of $62.63 \pm 9.39 \mathrm{~kg}$.

As the human gait is quite dynamic in the real-world often involving varying gait speeds, surfaces and surface inclinations, the proposed computational method was validated in uncontrolled indoor and outdoor environments. Three gait trials were randomly performed in the following scenarios (illustrated in Fig. 6): forward level-ground walking on a $20 \mathrm{~m}$ flat surface; forward level-ground walking on a rough surface (urban ground) along $30 \mathrm{~m}$; descending and ascending an inclined ground (approximately $10^{\circ}$ ) and a $10 \mathrm{~m}$ rough surface; and climbing a staircase of 8 steps with standard dimensions (a height of $17 \mathrm{~cm}$, depth of $31 \mathrm{~cm}$, and step width of $110 \mathrm{~cm}$ ). For each condition, the participants were asked to walk at three selfselected gait speeds: slow, normal, and fast.

\section{RESULTS}

A total of 5657 steps from both feet were analyzed to demonstrate the versatility and time-effectiveness of the adaptive computational method. We inspected 3522 controlled steps, 596 steps on flat surface, 572 steps on rough ground, and 644 steps on inclined ground, and 323 steps on staircases. This section presents the adaptability outcomes and performance metrics, such as the algorithm's precision and timing errors.

\section{A. Adaptability}

Adaptability is a key feature of the proposed computational method. Appendix II shows the gyroscope variability with sudden changes in speed, as well as the consequent variations in the adaptive thresholds $\left(M A X_{t h r}\right.$ and $\left.M I N_{t h r}\right)$ and adaptive ranges based on the stride (STRIDE_TIME) defined for each gait event (HS_range, FF_range, MMST_range, HO_range, $T O \_$range, $M M S W \_$range). The increase in gait speed results in higher values of angular velocity with shorter stride duration, supporting the need to update the thresholds of $M A X_{t h r}, M I N_{t h r}$ and STRIDE_TIME, respectively. By analyzing Appendix II, it is possible to conclude that the values of the adaptive thresholds (MAXthr and MINthr) increase or decrease when the magnitude of the maxima and minima are higher or lower, respectively, and the adaptive ranges, which are directly dependent on the value of the STRIDE_TIME (blue line in bottom view of Appendix II), change in accordance with these values.

The findings also highlight that the algorithm's adaptability provides a proper detection (orange line in top view of Appendix II) even when the foot angular velocity varies with changes in speed from $1.5 \mathrm{~km} / \mathrm{h}$ to $4.5 \mathrm{~km} / \mathrm{h}$ (controlled situation) and from $1 \pm 0.2 \mathrm{~m} / \mathrm{s}$ to $2 \pm 0.18 \mathrm{~m} / \mathrm{s}$ (real-life situations). The algorithm's adaptability can also address possible changes in the magnitude of the angular velocity, which may result from walking in overground (flat and rough ground) or on staircases.

The STRIDE_TIME calculation also contributes to the realtime estimation of stride time. STRIDE_TIME values were compared to the time between two consecutive HSs (tracked by the FSR placed on the heel) to determine the estimation error for different walking speeds (controlled situation) and surfaces (real-life situation). Table II lists the percentage of underestimated values (i.e., when the STRIDE_TIME was lower than the real stride time), the percentage of overestimated values (i.e., when the STRIDE_TIME was higher than the actual stride time), and the duration of these timing errors. The percentage values of these errors and the correct determinations of stride time sum to $100 \%$.

Overall, for the controlled situations the proposed approach more frequently determines a higher STRIDE_TIME than the 
actual value of the subjects, since the percentages of overestimations are higher than $50 \%$ (overestimated error > $59.1 \%)$. A similar finding can be observed with respect to the timing error since the overestimation $(<34.11 \pm 8.86 \mathrm{~ms})$ present higher errors than the underestimation $(<25.07 \pm 11.07 \mathrm{~ms})$. When the walking speed is variable, the adaptive STRIDE_TIME estimation exhibits similar time errors in terms of over and underestimation. These timing errors present a low significance considering that the magnitude of the stride time ranges from $1200 \mathrm{~ms}$ (walking speed $4.5 \mathrm{~km} / \mathrm{h}$ ) to $1750 \mathrm{~ms}$ (walking speed $1.5 \mathrm{~km} / \mathrm{h}$ ).

By analyzing the real-life situations in Table II, the findings suggest a higher occurrence of tuned estimations $(>27.5 \%)$ in the controlled situations $(>11.86 \%)$. This suggests that the adaptability point of STRIDE_TIME acted properly in real-life walking conditions that included changes in speed and surface. However, in terms of timing error, the proposed approach to estimate STRIDE_TIME performs similar in controlled and real-life situation, excepting stair climbing. In this walking condition, STRIDE_TIME was overestimated with a mean error of $56.01 \pm 15.68 \mathrm{~ms}$, i.e., the estimated value was higher than the actual value of $56.01 \pm 15.68 \mathrm{~ms}$. Although climbing stairs showed higher timing errors of the STRIDE_TIME, the magnitude of the error $56.01 \pm 15.68 \mathrm{~ms}$ was not quite significant when compared to the real value of stride time, which ranged from $1170 \mathrm{~ms}$ to $1350 \mathrm{~ms}$. For changing surfaces, the occurrence of overestimations $(<44.17 \%)$ was also more frequent than the occurrence of underestimations $(<37.82 \%)$.

TABLE II

UNDERESTIMATION AND OVERESTIMATION ERRORS OF STRIDE_TIME (\% OCCURRENCE AND TIMING ERROR). CORRECT ESTIMATIONS NOT REPORTED

\begin{tabular}{|c|c|c|c|c|c|}
\hline & \multirow{2}{*}{$\begin{array}{c}\text { Walking } \\
\text { condition }\end{array}$} & \multicolumn{2}{|c|}{ Underestimation Error } & \multicolumn{2}{|c|}{ Overestimation Error } \\
\hline & & $\%$ & ms & $\%$ & ms \\
\hline \multirow{5}{*}{ 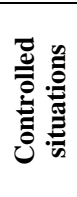 } & $1.5 \mathrm{~km} / \mathrm{h}$ & 24.54 & $25.07 \pm 11.07$ & 62.5 & $34.11 \pm 8.86$ \\
\hline & $2.5 \mathrm{~km} / \mathrm{h}$ & 27.16 & $17.8 \pm 10.68$ & 59.9 & $29.91 \pm 9.12$ \\
\hline & $3.5 \mathrm{~km} / \mathrm{h}$ & 24.53 & $14.64 \pm 7.95$ & 58.1 & $30.15 \pm 7.33$ \\
\hline & $4.5 \mathrm{~km} / \mathrm{h}$ & 27.05 & $13.57 \pm 8.85$ & 59.1 & $33.16 \pm 11.32$ \\
\hline & $\begin{array}{c}\text { Variable } \\
\text { speed }\end{array}$ & 40.94 & $30.09 \pm 6.82$ & 47.2 & $31.07 \pm 9.96$ \\
\hline \multirow{3}{*}{ 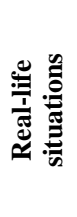 } & $\begin{array}{l}\text { Level- } \\
\text { ground }\end{array}$ & 28.33 & $19.59 \pm 12.33$ & 44.17 & $31.13 \pm 15.11$ \\
\hline & $\begin{array}{l}\text { Inclined } \\
\text { surfaces }\end{array}$ & 37.82 & $22.39 \pm 14.45$ & 39.90 & $23.0 \pm 14.83$ \\
\hline & $\begin{array}{l}\text { Climbing } \\
\text { stairs }\end{array}$ & 32.76 & $34.56 \pm 17.41$ & 39.66 & $56.01 \pm 15.68$ \\
\hline
\end{tabular}

\section{B. Performance metrics}

We investigated the accuracy, the percentage of occurrence and duration of delays and advances in the controlled (Table III) and real-life situations (Tables IV, V and VI) to assess the versatility and time-effectiveness of the algorithm. The timeeffectiveness was only inspected for correct detections. Timing errors greater than $100 \mathrm{~ms}$ (a critical duration for motor rehabilitation purposes) were considered as a misdetection. Appendix III presents the results of the statistical studies conducted to assess the performance of the proposed method.

We also assessed the operability of a state-of-the-art gait event detection algorithm (four-state hidden Markov model [19]) using the same dataset in an attempt to conduct a direct comparison with an existing method. We selected this detector, which relies on the same input data (angular velocity in the sagittal plane recorded by a foot-mounted gyroscope on healthy subjects) to the ones collected in the present work. Note that this comparison will be limited to the gait events determined by Mannini's algorithm [19], which consist of the HS, FF, HO and TO events. We also conducted a statistical analysis to strengthen this comparative analysis. The results of this analysis are presented in Appendix III.

By analyzing Table III, we verified that the proposed computational method is significantly accurate $(p=0.0812)$ for the detection of all events at distinct conditions (e.g., speed, slope, footwear or barefoot) in the controlled situation (accuracy $>95.06 \%$ ). The TO and MMSW events exhibited lower accuracy (accuracies of $95.95 \%$ and $95.06 \%$, respectively) due to the existence of local maxima and minima, respectively. On the other hand, the HS event was properly segmented in all the studied gait cycles (accuracy of 100\%). The high accuracy was consistent across different walking speeds and slopes, which suggests that the proposed adaptive tool can be applied to human gait analysis in real-life situations. The findings of the controlled situations also indicate that $\mathrm{HO}$ and MMST had a higher occurrence of delayed detections (30.80\% and $29.35 \%$, respectively), being detected with a mean delay of $22.71 \pm 21.07 \mathrm{~ms}$ and $36.54 \pm 13.25 \mathrm{~ms}$, respectively. Advanced detections were mainly observed for the $\mathrm{HO}$ $(18.62 \pm 9.63 \mathrm{~ms})$ and TO events $(14.38 \pm 12.83 \mathrm{~ms})$. The proposed algorithm showed significantly lower timing errors $(p=0.9314)$ and a lower occurrence of misdetection $(p=0.9953)$ than did the Mannini's algorithm. Additionally, this difference was more pronounced for the delayed detections namely, for the HO event (61.04 $\pm 10.81 \mathrm{~ms})$.

The findings presented in Tables IV, V and VI indicate that the proposed algorithm is significantly more accurate $(p>0.0526)$ with level-ground and inclined surfaces than in staircases $(p<0.05)$. However, with level-ground surfaces the timing errors were significantly different than $0 \mathrm{~ms}(p<0.05)$, in contrast to incline $(p>0.0942)$ and staircases $(p>0.151)$.

Considering the proposed computational method, HS was the most correctly detected event in the real-life walking situations (accuracy $>96.98 \%$ ) and was always correct in level-ground walking (accuracy of 100\%). On the other hand, MMSW was the least properly detected event (accuracy $>90.12 \%$ ) due to the existence of local maxima, which were particularly observed in two male subjects. For the remaining gait events, the algorithm performed similarly for the level-ground and inclined surfaces. Timing errors were more pronounced in the detection of MMST and HO events. In some analyzed gait cycles, the MMST event was detected earlier (mean advances $<22.2 \pm 067 \mathrm{~ms}$ ), whereas in other cases this event was detected with a mean delay lower than $18.79 \pm 3.2 \mathrm{~ms}$. In fact, this event was the earlier detected. This timing deviation may result from the algorithm's susceptibility to variations in stride, which causing delays and advances when the stride decreases or increases, respectively. HO was the most delayed gait event (mean delays $<26.39 \pm 4.67 \mathrm{~ms}$ ) due to possible instabilities of the signal during stance (not completely constant). The 
remaining gait events presented lower timing errors, which indicates that the algorithm can operate in a timely manner in normal real-life situations, such as stairs and inclined surfaces.

Moreover, the proposed method was more accurate (accuracy>96.98\%) in the detection of HS, FF, HO and TO events performed in the explored real-life situations than was Mannini's algorithm (accuracy>85.47\%). Mannini's method also demonstrated higher timing errors than the proposed method, which mainly consisted of the delayed detections of HS $(61.75 \pm 2.4 \mathrm{~ms})$ and $\mathrm{HO}$ events $(67.39 \pm 8.92 \mathrm{~ms})$, as well as the advanced detections of the HO event $(<52.35 \pm 4.67 \mathrm{~ms})$. Indeed, the proposed algorithm is significantly more accurate $(p>0.9925)$ and time-effective $(p>0.9314)$ in real-life situations than Mannini's method. Note that the timing errors disclosed in Tables III, IV, V and VI do not include the algorithm latency of $10 \mathrm{~ms}$ due to the filtering process.

TABLE III

ALGORITHMS PERFORMANCE IN CONTROLLED SITUATIONS

\begin{tabular}{|c|c|c|c|c|c|c|c|c|c|c|}
\hline & \multicolumn{5}{|c|}{ Proposed computational method } & \multicolumn{5}{|c|}{ Mannini's computational method [19] } \\
\hline & \multirow{2}{*}{$\begin{array}{c}\text { Accuracy } \\
(\%) \\
\end{array}$} & \multicolumn{2}{|r|}{ Delay } & \multicolumn{2}{|c|}{ Advance } & \multirow{2}{*}{$\begin{array}{c}\text { Accuracy } \\
(\%) \\
\end{array}$} & \multicolumn{2}{|c|}{ Delay } & \multicolumn{2}{|c|}{ Advance } \\
\hline & & $\%$ & ms & $\%$ & ms & & $\%$ & ms & $\%$ & ms \\
\hline $\mathbf{F F}$ & 99.37 & 6.23 & $4.36 \pm 5.06$ & 9.21 & $9.18 \pm 17.79$ & 92.37 & 9.16 & $52.76 \pm 20.67$ & 90.31 & $20.84 \pm 19.63$ \\
\hline MMST & 98.78 & 20.46 & $30.53 \pm 9.88$ & 7.92 & $15.31 \pm 5.52$ & - & - & - & - & - \\
\hline HO & 99.27 & 23.78 & $19.67 \pm 16.9$ & 11.02 & $13.75 \pm 9.59$ & 88.27 & 81.26 & $61.04 \pm 10.81$ & 16.0 & $24.72 \pm 12.03$ \\
\hline MMSW & 94.71 & 7.41 & $4.54 \pm 4.28$ & 0.29 & $1.75 \pm 4.45$ & - & - & - & - & - \\
\hline
\end{tabular}

TABLE IV

ALGORITHMS PERFORMANCE IN REAL-LIFE SITUATIONS: LEVEL-GROUND SURFACES

\begin{tabular}{|c|c|c|c|c|c|c|c|c|c|c|}
\hline & \multicolumn{5}{|c|}{ Proposed computational method } & \multicolumn{5}{|c|}{ Mannini's computational method [19] } \\
\hline & \multirow{2}{*}{$\begin{array}{c}\text { Accuracy } \\
(\%) \\
\end{array}$} & \multicolumn{2}{|r|}{ Delay } & \multicolumn{2}{|c|}{ Advance } & \multirow{2}{*}{$\begin{array}{c}\text { Accuracy } \\
(\%)\end{array}$} & \multicolumn{2}{|c|}{ Delay } & \multicolumn{2}{|c|}{ Advance } \\
\hline & & $\%$ & ms & $\%$ & ms & & $\%$ & ms & $\%$ & ms \\
\hline $\mathbf{F F}$ & 99.24 & 2.8 & $2.22 \pm 8.56$ & 1.6 & $1.19 \pm 9.46$ & 91.32 & 34.42 & $46.05 \pm 8.65$ & 63.40 & $23.15 \pm 2.56$ \\
\hline MMST & 91.04 & 23.5 & $8.63 \pm 12.5$ & 16.7 & $8.43 \pm 4.24$ & - & - & - & - & - \\
\hline HO & 96.18 & 30.8 & $26.39 \pm 4.7$ & 0 & 0.00 & 86.37 & 68.11 & $43.69 \pm 6.7$ & 31.40 & $37.52 \pm 5.61$ \\
\hline MMSW & 90.50 & 4.5 & $8.40 \pm 2.65$ & 0.3 & $5.3 \pm 0.80$ & - & - & - & - & - \\
\hline
\end{tabular}

TABLE V

ALGORITHMS PERFORMANCE IN REAL-LIFE SITUATIONS: INCLINED SURFACES

\begin{tabular}{|c|c|c|c|c|c|c|c|c|c|c|}
\hline & \multicolumn{5}{|c|}{ Proposed computational method } & \multicolumn{5}{|c|}{ Mannini's computational method [19] } \\
\hline & \multirow{2}{*}{$\begin{array}{c}\text { Accuracy } \\
(\%)\end{array}$} & \multicolumn{2}{|r|}{ Delay } & \multicolumn{2}{|c|}{ Advance } & \multirow{2}{*}{$\begin{array}{c}\text { Accuracy } \\
(\%)\end{array}$} & \multicolumn{2}{|c|}{ Delay } & \multicolumn{2}{|c|}{ Advance } \\
\hline & & $\%$ & ms & $\%$ & ms & & $\%$ & $\mathbf{m s}$ & $\%$ & ms \\
\hline HS & 99.82 & 0 & $0.0 \pm 0.0$ & 2.3 & $0.71 \pm 2.45$ & 92.57 & 1.44 & $61.75 \pm 2.4$ & 98.56 & $11.50 \pm 7.60$ \\
\hline FF & 99.82 & 0 & $0.0 \pm 0.0$ & 2.7 & $1.43 \pm 1.98$ & 91.10 & 16.85 & $23.36 \pm 3.21$ & 82.79 & $19.49 \pm 4.78$ \\
\hline MMST & 91.87 & 22.7 & $18.79 \pm 3.2$ & 18.67 & $2.14 \pm 0.67$ & - & - & - & - & - \\
\hline HO & 96.17 & 28.9 & $16.43 \pm 12.3$ & 1.6 & $0.71 \pm 0.56$ & 85.47 & 85.89 & $38.11 \pm 6.54$ & 13.80 & $48.32 \pm 4.81$ \\
\hline
\end{tabular}

TABLE VI

ALGORITHMS PERFORMANCE IN REAL-LIFE SITUATIONS: STAIRS

\begin{tabular}{|c|c|c|c|c|c|c|c|c|c|c|}
\hline & \multicolumn{5}{|c|}{ Proposed computational method } & \multicolumn{5}{|c|}{ Mannini's computational method [19] } \\
\hline & \multirow{2}{*}{$\begin{array}{c}\text { Accuracy } \\
(\%)\end{array}$} & \multicolumn{2}{|r|}{ Delay } & \multicolumn{2}{|c|}{ Advance } & \multirow{2}{*}{$\begin{array}{c}\text { Accuracy } \\
(\%)\end{array}$} & \multicolumn{2}{|c|}{ Delay } & \multicolumn{2}{|c|}{ Advance } \\
\hline & & $\%$ & ms & $\%$ & $\mathbf{m s}$ & & $\%$ & ms & $\%$ & ms \\
\hline HS & 96.98 & 0 & $0.0 \pm 0.0$ & 2.56 & $2.51 \pm 1.56$ & 90.46 & 10.6 & $44.91 \pm 5.2$ & 89.42 & $18.88 \pm 2.45$ \\
\hline FF & 96.78 & 0 & $0.0 \pm 0.0$ & 2.4 & $1.43 \pm 1.98$ & 89.43 & 9.0 & $58.88 \pm 6.71$ & 91.0 & $23.59 \pm 1.45$ \\
\hline MMST & 92.79 & 27.41 & $16.79 \pm 1.2$ & 12.67 & $22.2 \pm 0.67$ & - & - & - & - & - \\
\hline HO & 93.98 & 24.6 & $23.6 \pm 7.3$ & 0 & $0.0 \pm 0.0$ & 81.67 & 80.0 & $67.39 \pm 8.92$ & 18.57 & $52.35 \pm 4.67$ \\
\hline TO & 95.89 & 0 & $0.0 \pm 0.0$ & 3.3 & $6.43 \pm 5.7$ & 85.47 & 79.59 & $24.94 \pm 2.60$ & 20.41 & $45.97 \pm 4.60$ \\
\hline MMSW & 90.79 & 2.63 & $2.63 \pm 3.16$ & 0 & $0.0 \pm 0.0$ & - & - & - & - & - \\
\hline
\end{tabular}




\section{DISCUSSION}

A real-time and adaptive computational method for assessing human gait events was presented and validated in controlled and real-life walking situations using repeated measures of healthy gait patterns. The novelty of this adaptive algorithm lies in using a single kinematic measure to detect six gait events (HS, FF, MMST, HO, TO, and MMSW) in real-life scenarios, which include variations in gait speeds and surfaces. The single axis of a gyroscope placed on the instep of each foot provides sufficient information for the segmentation of human gait events at these conditions. This single measurement point simplifies the computational load of the gait analysis and enables its integration for daily and repeated use.

The computational method proposed in this paper was coupled to a threshold-based structure where an FSM detects the events and, in parallel, updates the thresholds used in the heuristic decision rules. The heuristic decision rules involve the gyroscope information that varies with variations in speed and surface. Consequently, the algorithm's adaptability proved to be a key feature for the successful application of the proposed gait event detection system in real-life situations, and it enabled the algorithm to handle inter-subject and inter-step variability. This feature makes this computational method a potential benchmark approach for real-time human gait segmentation.

The proposed gait event detection system endows different strategies to reduce latency. The use of a wired connection in the wearable sensory system and the processing of only one axis of kinematic data in a high-performance central processing unit are factors that reduce the latency. Additionally, we applied a $1^{\text {st }}$ order low-pass filter that is computationally light.

The proposed algorithm was significantly accurate (accuracy equal to $100 \%, p>0.0526$ ) in most of the investigated walking situations (controlled situations, level-ground and inclined surfaces). In addition, the proposed method is a time-effective tool for real-life situations, as shown by delays that were significantly closer to $0 \mathrm{~ms}(p>0.0663)$.

In comparison with the literature regarding real-time gait event detection based on gyroscope signals, the proposed computational method was able to conduct a more holistic gait segmentation by detecting six gait events instead of only detecting HS and TO events (the most commonly detected events). Moreover, this work is advantageously when compared with similar studies (i.e., using heuristic rules based on foot angular velocity) conducted in controlled situations and on level-ground surfaces. Bejarano et al. [3] reported delays of $69.6 \pm 15.1 \mathrm{~ms}$ and $7.8 \pm 25.6 \mathrm{~ms}$ for $\mathrm{HS}$ and TO, respectively whereas the proposed tool has shown to be more time-effective (HS: $6.28 \pm 12.03 \mathrm{~ms}$ and TO: $5.82 \pm 15.95 \mathrm{~ms}$ ), mainly in HS detection. Similarly, delayed detection was reported by Gowanda et al. [17] and Lee et al. [18] for HS (100 ms and 19 $\mathrm{ms}$, respectively) and TO (100 ms and $8 \mathrm{~ms}$, respectively) on level-ground walking.

The performance of the presented algorithm also matches that of the current state-of-the-art real-time HS and TO detection in real-life situations. To the best knowledge of the authors, there has been no study in the literature that was able to detect the six gait events assessed in the present study in both inclined surfaces and staircases. Catalfamo et al. [6] reported that their heuristic rules were able to segment the HS and TO events (accuracy of 98\%) on inclined surfaces (indoor and outdoor environments) with a delay of $25 \mathrm{~ms}$ and an advance of 75 ms. Our adaptive method was shown to be more timeeffective (timing errors less than $6.43 \pm 5.7 \mathrm{~ms}$ ), and similarly accurate (accuracy of $99.82 \%$ and $97.13 \%$ for HS and TO, respectively). The algorithm's adaptability also contributed to positive findings for gait cycles performed on staircases. Our approach produced favorable performance in terms of accuracy $(\mathrm{HS}=96.98 \%>95.5 \%$; TO $=95.89 \%>93.1 \%)$ and timeeffectiveness $(\mathrm{HS}=0.0 \pm 0.0 \mathrm{~ms}<11 \pm 18 \mathrm{~ms} ; \mathrm{TO}=6.43 \pm 5.7 \mathrm{~ms}<$ $35 \pm 20 \mathrm{~ms}$ ) as compared to study [16].

Furthermore, through a direct comparison with the Hidden Markov Models proposed by Mannini et al. [19] using the same dataset, we verified that the proposed approach performs advantageously in the detection of HS (delays of 61.75>6.28 $\mathrm{ms}$; advances of 19.77>10.25ms), FF (delays of $58.88>4.36 \mathrm{~ms}$; advances of 23.59>9.18 ms), $\mathrm{HO}$ (delays of $67.39>26.39 \mathrm{~ms}$; advances of $52.35>11.02 \mathrm{~ms}$ ), and TO (delays of 24.94>5.82 ms; advances of $28.09>18.24 \mathrm{~ms}$ ). For controlled and real-life situations, the proposed adaptive rule-based method was significantly time-effective $(p>0.9314)$ and more accurate (accuracy $=96.98 \%>85.47 \%, \quad p>0.9925)$ than the machine learning algorithm proposed by Mannini et al. [19]. This benchmark analysis highlights the benefits of the proposed computational method for the gait event detection field.

Moreover, the algorithm was shown to be robust in barefoot and footwear conditions, even when different types (size, shape, and height of sole) of shoes were worn. This finding highlights the versatility of the proposed tool for different user's foot conditions in opposition to the force-based sensors, whose performance relies on foot size and shape.

The proposed method was also able to determine the stride time in real-time, a temporal gait parameter commonly observed in human gait diagnosis. For both controlled and reallife situations, the stride time was more overestimated $(62.5 \%$ of occurrences) than underestimated (40.94\% of occurrences). However, this effect was observed less under changes in surface and speed, highlighting the suitability of the proposed computational method for real-life situations. Moreover, the exhibited timing errors were lower when compared to the actual value of the stride time. Overall, the findings indicate that the computational method can properly update the stride time.

The lower computational load, together with the significantly improved performance of the adaptive computational method, increases the potential application of the proposed method as a quantitative and computational benchmark for assessing human gait. It is also shown to be suitable for providing human gait information in real-time by outputting lower timing errors, which are smaller than the reaction times of healthy voluntary muscle activities (128 $\pm 3 \mathrm{~ms}$ ) [32]. As the delay presented by the developed tool is considerably lower than the response time of human physiological structures, the integration of this algorithm in real-time control of human gait is also feasible.

Nonetheless, improvements can be performed to mitigate the different factors that could lead to timing deviations and 
misdetections. For instance, the detection of local extrema should be more tuned to minimize the occurrence of misdetections or timing errors in the MMSW and TO events to address the occurrence of local maxima and minima, respectively, close to the global extrema. For MMST, the detection method must be more robust to the variations of the stride time to avoid timing errors when this gait metric decreases or increases. Finally, note that the validation presented in this work only considered straight-line walking.

\section{CONCLUSION}

The proposed gait event detection system was shown to be an accurate, time-effective, low-cost, wearable, lowcomputation strategy for real-time gait analysis, which can be used either in gait assessment or rehabilitation tasks. The adaptability introduced in this tool provides more accurate gait analysis in different walking conditions and enables more robust accommodation of sporadic perturbations. These aspects, combined with the reduced computational load and simple usage, makes this detection system suitable as a quantitative benchmark of human locomotion.

Future work is aimed at enhancing the threshold-based algorithm to reduce the effects of local extrema and to apply this detection system in the control of a lower-limb orthosis. Challenges also include the validation of this algorithm with neurological subjects in non-assisted and assisted gait conditions. In the latter case, predictive techniques can also be explored to tune the assistance delivered by robotic devices with the user's gait pattern.

\section{ACKNOWLEDGMENT}

The present work involved the collaboration of Andrea Mannini (BioRobotics Institute, Italy), who executed the Hidden Markov Models presented in [19] with the dataset collected in this work.

\section{REFERENCES}

[1] J. Taborri, E. Palermo, S. Rossi, and P. Cappa, "Gait partitioning methods: A systematic review," Sensors (Switzerland), vol. 16, no. 1, pp. 40-42, 2016.

[2] N. Abaid, P. Cappa, E. Palermo, M. Petrarca, and M. Porfiri, "Gait Detection in Children with and without Hemiplegia Using Single-Axis Wearable Gyroscopes," PLoS One, vol. 8, no. 9, pp. 1-8, 2013.

[3] N. C. Bejarano, E. Ambrosini, a. Pedrocchi, G. Ferrigno, M. Monticone, and S. Ferrante, "An adaptive real-time algorithm to detect gait events using inertial sensors," IFMBE Proc., vol. 41, pp. 1799-1802, 2014.

[4] A. U. Alahakone, S. M. N. A. Senanayake, and C. M. Senanayake, "Smart wearable device for real time gait event detection during running," IEEE Asia-Pacific Conf. Circuits Syst. Proceedings, APCCAS, pp. 612-615, 2010.

[5] S. Lambrecht, A. Harutyunyan, K. Tanghe, M. Afschrift, J. De Schutter, and I. Jonkers, "Real-time gait event detection based on kinematic data coupled to a biomechanical model," Sensors (Switzerland), vol. 17, no. 4, 2017.

[6] Catalfamo, P, S. Ghoussayni, and D. Ewins, "Gait Event Detection on Level Ground and Incline Walking Using a Rate Gyroscope," Sensors, vol. 10 , no. Ic, pp. 5683-5702, 2011

[7] A. M. Sabatini, C. Martelloni, S. Scapellato, and F. Cavallo, "Assessment of walking features from foot inertial sensing," IEEE Trans. Biomed. Eng., vol. 52, no. 3, pp. 486-494, 2005.

[8] M. Hanlon and R. Anderson, "Real-time gait event detection using wearable sensors.," Gait Posture, vol. 30, no. 4, pp. 523-7, Nov. 2009.
[9] N. C. Bejarano, E. Ambrosini, A. Pedrocchi, G. Ferrigno, M. Monticone, and S. Ferrante, "A novel adaptive, real-time algorithm to detect gait events from wearable sensors," IEEE Trans. Neural Syst. Rehabil. Eng., vol. 23, no. 3, pp. 413-422, 2015.

[10] J. Rueterbories, E. G. Spaich, and O. K. Andersen, "Gait event detection for use in FES rehabilitation by radial and tangential foot accelerations," Med. Eng. Phys., vol. 36, no. 4, pp. 502-508, 2014.

[11] M. S. H. Aung et al., "Automated detection of instantaneous gait events using time frequency analysis and manifold embedding," IEEE Trans. Neural Syst. Rehabil. Eng., vol. 21, no. 6, pp. 908-916, 2013.

[12] I. P. I. Pappas, M. R. Popovic, T. Keller, V. Dietz, and M. Morari, "A reliable gait phase detection system," IEEE Trans. Neural Syst. Rehabil. Eng., vol. 9, no. 2, pp. 113-125, 2001.

[13] R. C. González, A. M. López, J. Rodriguez-Uría, D. Álvarez, and J. C. Alvarez, "Real-time gait event detection for normal subjects from lower trunk accelerations," Gait Posture, vol. 31, pp. 322-325, 2010.

[14] S. Khandelwal and N. Wickström, "Evaluation of the performance of accelerometer-based gait event detection algorithms in different real-world scenarios using the MAREA gait database," Gait Posture, vol. 51, pp. 8490, 2017

[15] M. A. Azhar, D. Gouwanda, and A. A. Gopalai, "Development of an Intelligent Real - time Heuristic - based Algorithm to Identify Human Gait Events," pp. 573-576, 2014.

[16] P. C. Formento, R. Acevedo, S. Ghoussayni, and D. Ewins, "Gait event detection during stair walking using a rate gyroscope," Sensors (Basel)., vol. 14, no. 3, pp. 5470-5485, 2014.

[17] D. Gouwanda and A. A. Gopalai, "A robust real-time gait event detection using wireless gyroscope and its application on normal and altered gaits," Med. Eng. Phys., vol. 37, pp. 219-225, 2015.

[18] J. K. Lee and E. J. Park, "Quasi real-time gait event detection using shankattached gyroscopes," Med. Biol. Eng. Comput., vol. 49, pp. 707-712, 2011.

[19] A. Mannini, V. Genovese, A. M. Sabatini, and S. Member, "Online Decoding of Hidden Markov Models for Gait Event Detection Using FootMounted Gyroscopes," IEEE J. Biomed. Heal. INFORMATICS, vol. 18, no. 4, pp. 1122-1130, 2014.

[20] J. Barth et al., "Stride segmentation during free walk movements using multi-dimensional subsequence dynamic time warping on inertial sensor data," Sensors (Switzerland), vol. 15, no. 3, pp. 6419-6440, 2015.

[21] M. Goršič et al., "Online phase detection using wearable sensors for walking with a robotic prosthesis," Sensors (Switzerland), vol. 14, no. 2, pp. 2776-2794, 2014.

[22] D. Kotiadis, H. J. Hermens, and P. H. Veltink, "Inertial Gait Phase Detection for control of a drop foot stimulator. Inertial sensing for gait phase detection.," Med. Eng. Phys., vol. 32, no. 4, pp. 287-297, 2010.

[23] F. A. Storm, C. J. Buckley, and C. Mazzà, "Gait event detection in laboratory and real life settings: Accuracy of ankle and waist sensor based methods," Gait Posture, vol. 50, pp. 42-46, 2016.

[24] T. Seel, "Learning Control and Inertial Realtime Gait Analysis in Biomedical Applications,” Technischen Universität Berlin, 2016.

[25] J. Figueiredo, C. Ferreira, C. P. Santos, J. C. Moreno, and L. P. Reis, "RealTime Gait Events Detection During Walking of Biped Model and Humanoid Robot Through Adaptive Thresholds," IEEE Int. Conf. Auton. Robot Syst. Compet., pp. 1-6, 2016.

[26] P. Félix, J. Figueiredo, C. P. Santos, and J. C. Moreno, "Adaptive real-time tool for human gait event detection using a wearable gyroscope," 20th Int. Conf. Climbing Walk. Robot. Support Technol. Mob. Mach. (CLAWAR 2017), pp. 1-9, 2017.

[27] I. González, J. Fontecha, R. Hervás, and J. Bravo, "An Ambulatory System for Gait Monitoring Based on Wireless Sensorized Insoles," Sensors, vol. 15, no. 7, pp. 16589-16613, 2015.

[28] D. a Bruening and S. T. Ridge, "Automated event detection algorithms in pathological gait.," Gait Posture, vol. 39, no. 1, pp. 472-7, 2014.

[29] I. P. I. Pappas, T. Keller, S. Mangold, M. R. Popovic, V. Dietz, and M. Morari, "A Reliable Gyroscope-Based Gait-Phase Detection Sensor Embedded in a Shoe Insole," IEEE Sens. J., vol. 4, no. 2, pp. 268-274, 2004.

[30] A. Ferrari, P. Ginis, M. Hardegger, F. Casamassima, L. Rocchi, and L. Chiari, "A Mobile Kalman-Filter Based Solution for the Real-Time Estimation of Spatio-Temporal Gait Parameters," IEEE Trans. Neural Syst. Rehabil. Eng., vol. 24, no. 7, pp. 764-773, 2016.

[31] J. Perry, GAIT Pathological Function. 1992.

[32] B. L. Day et al., "Delay in the execution of voluntary movement by electrical or magnetic brain stimulation in intact man.," Brain, vol. 112, pp. 649-663, 1989. 
APPENDIX I
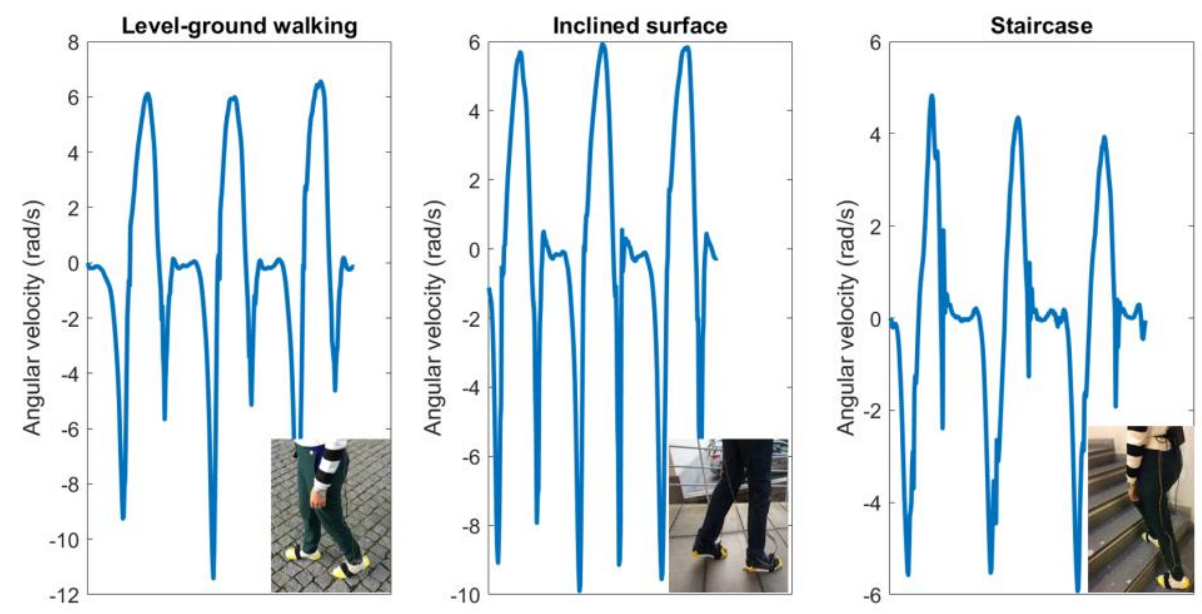

Foot angular velocity along sensor's z-axis (moves relatively to the sagittal plane) measured at different ground facets: levelground, inclined surface $\left(10^{\circ}\right)$, and staircase.

APPENDIX II
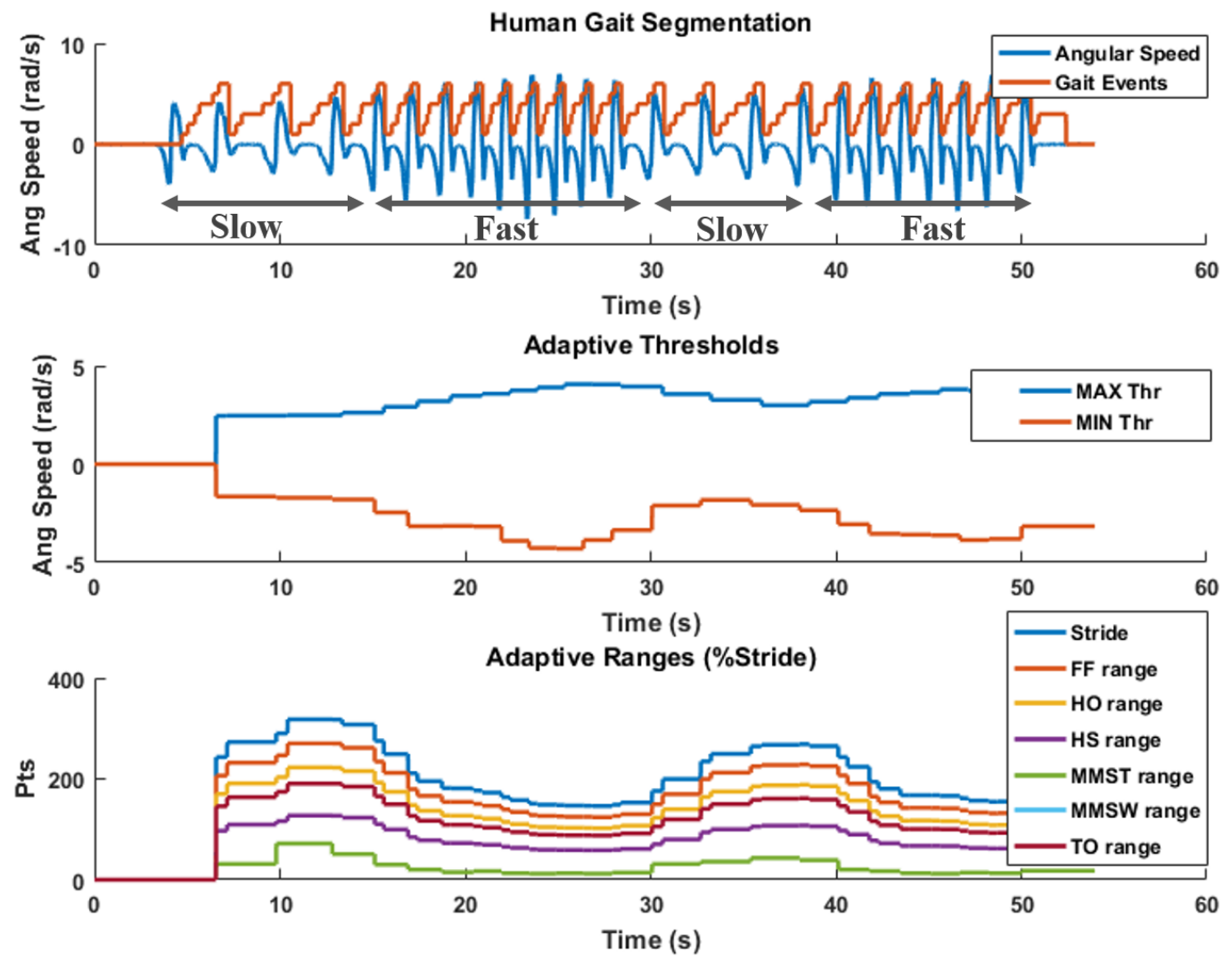

Human gait detection in one subject (top view), walking at distinct speeds, with representation of adaptive thresholds (middle view) and adaptive ranges (bottom view) changing during the trial. 


\section{APPENDIX III}

\begin{tabular}{llll||ccc}
\hline \hline \multirow{2}{*}{ Walking Situation } & \multicolumn{2}{c|}{ Statistical performance of proposed method } & \multicolumn{3}{c}{ Proposed method $\boldsymbol{v s}$ Mannini's method } \\
\cline { 2 - 7 } & Accuracy & Delay & Advance & Accuracy & Delay & Advance \\
\hline Controlled situations & $p=0.0812$ & $p<0.05$ & $p<0.05$ & $p=0.9953$ & $p=0.9314$ & $p=0.9758$ \\
\hline Level-ground & $p=0.0639$ & $p=0.0663$ & $p<0.05$ & $p=0.9979$ & $p=0.9889$ & $p=0.9945$ \\
\hline Inclined & $p=0.0526$ & $p=0.0942$ & $p=0.1023$ & $p=0.9976$ & $p=0.9732$ & $p=0.9480$ \\
\hline Stairs & $p<0.05$ & $p=0.151$ & $p=0.1807$ & $p=0.9925$ & $p=0.9946$ & $p=0.9866$ \\
\hline \hline
\end{tabular}

Results of two statistical analyses conducted with a significance level of 5\%. In the first analysis, we statistically investigate the performance of the proposed method by testing its accuracy and time-effectiveness relatively to the desired performance, i.e., $100 \%$ and zero ms, respectively. In the second analysis, we performed a statistical study to increase the strength of the comparative analysis between the proposed computational method and the Mannini's method. For this purpose, we conducted two statistical tests with the following hypothesis; first, the accuracy of the proposed algorithm is greater than the one reached by the Mannini's method; second, the delay and advance times of the proposed algorithm are lower than the one reached by the Mannini's method. 